\section{THYROPET Study: Is It Biology or Technology That Is the Issue?}

TO THE EDITOR: We read with interest the results of the recently published THYROPET study (1), a prospective multicenter diagnostic cohort study testing the hypothesis that a recombinant human thyroid-stimulating hormone (rh-TSH)-stimulated ${ }^{124}$ I PET/ CT scan can identify patients with a negative thyroxine withdrawal (T4WD) posttherapy ${ }^{131}$ I scan and avoid futile treatment in patients with suspected recurrence of differentiated thyroid carcinoma. The trial was terminated prematurely because of a high number of falsenegative rh-TSH-stimulated ${ }^{124}$ I PET/CT scans, which would preclude potential therapeutic benefit from ${ }^{131}$ I therapy. Although this may be interpreted as a rebuttal of the theranostic approach and affirmation of the established practice of empiric ${ }^{131} \mathrm{I}$, we agree with the authors' conclusion that "124I PET/CT remains the most rational strategy to reduce futile ${ }^{131}$ I therapies" despite this statement's apparent conflict with their actual study findings. The explanation of this result will be critical to guide the use of ${ }^{124}$ I PET/CT for management of advanced thyroid cancer.

In particular, we would like to focus on the very different clinical implications arising from the possible technical and biologic explanations for the false-negative rh-TSH-stimulated ${ }^{124}$ I results. Radioiodine imaging is a classic theranostic investigation, whereby the distribution of iodine uptake and retention within tumor is used to predict the response to a therapeutic administered activity of ${ }^{131} \mathrm{I}$. A key advantage of ${ }^{124} \mathrm{I}$ PET/CT imaging is the opportunity to perform prospective dosimetry to more accurately predict this response (2). In this context, it is highly unlikely that a necessarily faint focus of uptake below the resolution of ${ }^{124}$ I PET but visible on post- ${ }^{131}$ I imaging would deliver a clinically meaningful dose of radiation. Thus, if technical differences were the only explanation for the high false-negative rate of ${ }^{124}$ I PET/CT, then it remains an appropriate screening investigation for this indication as the risks of high-dose ${ }^{131}$ I therapy are likely to outweigh the modest benefits due to unfavorable radiation dosimetry.

However, a recently published phantom study (3) confirms that there is no appreciable technical difference in detectability for even small spheres $(<10 \mathrm{~mm})$ at this administered activity $(74 \mathrm{MBq}$ of ${ }^{124}$ I) on scanners using point-spread function model-based resolution recovery and time-of-flight technology. We note that some but not all THYROPET study centers had time-of-flight technology, and thus, knowledge of the number of patients imaged using this protocol is necessary to better interpret the results of this study. Different imaging times are another potential technical explanation for uptake that was seen on delayed posttherapy scans $(5 \mathrm{~d})$ but missed on early (24 h) ${ }^{124}$ I imaging (4). However, this is unlikely to be relevant because of the dual-time-point 24- and 96-h acquisitions utilized in the THYROPET study.

In contrast, it remains plausible that the false-negative ${ }^{124} \mathrm{I}$ PET/CT scans reflect different biologic tumor responses to TSH stimulation from rh-TSH and thyroxine withdrawal. The au-

COPYRIGHT (C 2017 by the Society of Nuclear Medicine and Molecular Imaging. thors appropriately discuss this possibility with numerous published intrapatient case studies. We also note that in the subset of THYROPET cases, there was one patient who underwent ${ }^{124} \mathrm{I}$ PET/CT after both rhTSH and T4WD, and this patient had 23 times greater ${ }^{124}$ I retention after T4WD. If this finding were confirmed in a large, prospective, intrapatient ${ }^{124}$ I study, there would be profound clinical implications for the management of metastatic thyroid cancer, given the widespread use of off-label rh-TSH stimulation for both diagnostic ${ }^{124} \mathrm{I}$ and therapeutic ${ }^{131} \mathrm{I}$ therapy in this setting.

We believe it is more plausible that biologic differences between rh-TSH and T4WD stimulation of recurrent thyroid cancer explain the THYROPET results with significant clinical impact. To the extent that technical factors associated with ${ }^{124} \mathrm{I}$ imaging explain the THYROPET study findings, we believe that they are unlikely to affect its role as a theranostic test in this setting.

\section{REFERENCES}

1. Kist JW, de Keizer B, van der Vlies M, et al. ${ }^{124}$ I PET/CT to predict the outcome of blind ${ }^{131} \mathrm{I}$ treatment in patients with biochemical recurrence of differentiated thyroid cancer: results of a multicenter diagnostic cohort study (THYROPET). J Nucl Med. 2016;57:701-707.

2. Wierts R, Brans B, Havekes B, et al. Dose-response relationship in differentiated thyroid cancer patients undergoing radioiodine treatment assessed by means of ${ }^{124}$ I PET/CT. J Nucl Med. 2016;57:1027-1032.

3. Beijst C, Kist JW, Elschot M, et al. Quantitative comparison of ${ }^{124} \mathrm{I}$ PET/CT and ${ }^{131}$ I SPECT/CT detectability. J Nucl Med. 2016;57:103-108.

4. Freudenberg LS, Jentzen W, Müller SP, Bockisch A. Disseminated iodine-avid lung metastases in differentiated thyroid cancer: a challenge to ${ }^{124}$ I PET. Eur $J$ Nucl Med Mol Imaging. 2008;35:502-508.

David A. Pattison*
Rodney J. Hicks
*Peter MacCallum Cancer Centre
305 Grattan St.
Melbourne, Victoria, Australia 3000
E-mail: david.pattison@petermac.org

Published online Aug. 18, 2016.

DOI: $10.2967 /$ jnumed.116.181420

REPLY: Thank you for the opportunity to respond to the letter to the editor by Pattison et al. regarding our article presenting the results of the THYROPET study (1). That study showed that after preparation with recombinant human thyroid-stimulating hormone (rh-TSH), ${ }^{124}$ I PET/CT could not predict the outcome of the posttherapy ${ }^{131}$ I scan after thyroid hormone withdrawal (THW) in patients with suspected recurrence of differentiated thyroid cancer. We discussed several factors potentially causing these disappointing results; both technical issues regarding ${ }^{124} \mathrm{I}$ PET/CT acquisition and biologic matters were put forward.

Pattison et al. argue that the biologic explanations are most probably causing the false-negative ${ }^{124}$ I PET/CT scans. We fully agree that the difference in the method of preparation on the ${ }^{124}$ I PET/CT (after rhTSH injections) and the ${ }^{131}$ I therapy with posttherapy scanning (after THW) is a likely cause of the ${ }^{124}$ I PET/CT being false-negative. Data from ours and other studies support this hypothesis (1-3). Confirmation 